\title{
Nonlinear Optical Properties of Some Newly Developed Crystals for Measurement of Ultrafast Laser Pulses
}

\author{
Kamal Hussain and Pathik Kumbhakar \\ Department of Physics, National Institute of Technology-Durgapur, \\ Mahatma Gandhi Avenue, Durgapur-713209, West Bengal, India
}

Received on 16 September, 2006

\begin{abstract}
Second harmonic generation (SHG) in nonlinear optical crystal is well-established technique for the measurement of the pulse-width of ultrafast femtosecond laser pulses. However, only selected nonlinear crystalline materials are suited for this purpose due to the limitations in transparency cut-off, limited phase-matching wavelength range, and large difference between the group velocities of the fundamental and harmonic waves. It is found that some newly discovered crystals, such as $\mathrm{CsLiB}_{6} \mathrm{O}_{10}(\mathrm{CLBO}), \mathrm{K}_{2} \mathrm{Al}_{2} \mathrm{~B}_{2} \mathrm{O}_{7}$ (KABO), $\mathrm{Li}_{2} \mathrm{~B}_{4} \mathrm{O}_{7}$ (LB4), and $\mathrm{KBe}_{2} \mathrm{BO}_{3} \mathrm{~F}(\mathrm{KBBF})$ have some advantageous characteristics for use in ultrafast nonlinear optical applications. Here we have presented several linear and nonlinear optical parameters including phase-matching angle, angular and spectral acceptance bandwidths, walk-off angle, nonlinear coupling coefficient, and group-velocity mismatch for generation of second harmonic of ultrafast laser radiations by employing type-I and type-II SHG techniques in these crystals.
\end{abstract}

Keywords: Second harmonic generation; Vacuum ultraviolet generation; Ultrafast pulse Measurement; Nonlinear optical crystal

\section{INTRODUCTION}

The nonlinear optical (NLO) frequency conversion technique continues to be the best choice for generation of tunable coherent ultraviolet (UV) radiation and also second harmonic generation (SHG) in a NLO crystal is a simplest and widely used technique for characterization of broadband ultrafast laser pulses [1-4] generated via either noncollinear optical parametric amplification (NOPA) or by some other methods. In view of the transparency cut-off near the UV range and limited phase-matching (PM) range, most NLO materials are unsuitable for use in conversion to UV and thus their use for characterization of broadband UV-visible pulses becomes limited. Ultrafast optical pulses can provide high conversion efficiency due to their high peak power, but in this case the additional limitation is the temporal walk-off caused by different group velocities of the fundamental and the second harmonic radiations. This walk-off sets a limit on the interaction length and consequently thin samples should be used which further limits the conversion efficiency. The ideal crystal for UV ultrafast NLO application must have a small group velocity difference between the fundamental and harmonic waves and a large nonlinearity, broadband phase-matching, and must have no absorption at both the fundamental and harmonic wavelengths. It is favorable if the crystal is transparent at twice the harmonic frequency to avoid two-photon absorption. Recently, several borate based new NLO crystals have been discovered [5-9]. The special performances of these borate-based crystals are their capability to broaden the range of harmonic generation even in VUV spectral region in some newly discovered crystals. However, the detailed investigations on potentialities of these newly developed crystals in different ultrafast NLO applications remains unexplored. Here we have considered investigating numerically NLO properties of some potential crystals including $\beta$ $\mathrm{BaB}_{2} \mathrm{O}_{4}$ (BBO), $\mathrm{CsLiB}_{6} \mathrm{O}_{10}$ (CLBO), $\mathrm{K}_{2} \mathrm{Al}_{2} \mathrm{~B}_{2} \mathrm{O}_{7}$ (KABO),
$\mathrm{Li}_{2} \mathrm{~B}_{4} \mathrm{O}_{7}$ (LB4), and $\mathrm{KBe}_{2} \mathrm{BO}_{3} \mathrm{~F}$ (KBBF), for their capability to generate SHG wavelength in the deep-UV region including in the VUV region. The optical transparency ranges of BBO, CLBO, KABO, LB4, and KBBF crystals are 188$3500,175-2750,180-3600,160-3500$, and 155-3780 nm, respectively [5-9]. Among these crystals, BBO is widely used in different ultrafast NLO applications and others are not so popular, till yet. Here we have demonstrated some basic NLO properties of these crystals including phase matching angles, acceptance angle, acceptance wavelength, and walk-off angle. The values of group-velocity mismatch $\left(\Delta_{v g}\right)$ for the generation of UV and VUV laser radiation by type-I and type-II SHG in these crystals have also been reported. It is very much important to know the value of $\Delta_{v g}$ for NLO interactions with ultrafast laser pulses. However, it has been found that some of the newly discovered crystals have prospective properties for frequency conversion with ultrafast femtosecond laser radiation in UV spectral region.

\section{HARMONIC GENERATION FROM ULTRASHORT LASER PULSES}

The simplest method for obtaining the laser radiation in UV and deep-UV spectral region is the SHG of UV-visible radiation in a NLO media. In a suitable NLO crystals a frequency doubled $(2 \omega)$ optical radiation is generated, when two optical radiations of frequency $\omega$, having the same or orthogonal polarizations incident on it. Energy transfer from the input to the generated radiation becomes optimum when the phase velocities of the interacting radiations become same i.e. in the phase matching condition [9]. The wave dispersion results in group velocity mismatch in the creation of harmonic wave due to the two different group velocities of the fundamental and harmonic waves. For efficient conversion of energies from the fundamental to the harmonic radiation, both group velocity and phase velocity matching are required to achieve simul- 
taneously. In practical situation this is impossible since the group velocity indices exhibit stronger dispersion than the refractive indices. Due to group velocity mismatch, the fundamental and harmonic pulses propagate with different velocities inside the crystal and this may lead to temporal walk-off between the interacting radiations. This effect is detrimental to achieve large gain in SHG process as it reduces the effective interaction length of the crystal. For maximizing the conversion efficiency a reasonable large size crystal is required, but too large size is useless because only a small portion of the crystal becomes effective due to presence of spatial as well as temporal walk-off effects. The effective interaction length, $L_{e f f}$, is calculated using the following relation,

$$
L_{e f f}=T / \Delta_{v g} .
$$

Where, $T=$ temporal width of the fundamental pulse. For type-I (o+o-e) interaction $\Delta_{v g}=1 /\left(u_{1}^{-1}-u_{3}^{-1}\right), u_{1}$ and $u_{3}$ are group velocities of the o-polarized input $\left(\lambda_{1}\right)$ and the e-polarized generated $\left(\lambda_{3}\right)$ waves, respectively. For typeII (o+e-e) interaction $\Delta_{v g}=1 /\left(u_{1}^{-1}-u_{3}^{-1}\right), u_{1}$ and $u_{3}$ are group velocities of the o (or e)-polarized input $\left(\lambda_{1}\right)$ and the e-polarized generated $\left(\lambda_{3}\right)$ waves, respectively. Several NLO properties viz. for generation $400 \mathrm{~nm}$ laser radiation by typeI SHG in BBO, CLBO, KABO, LB4, and KBBF crystals from Ti:sapphire fundamental $800 \mathrm{~nm}$ laser radiation have been presented in Table 1. The methods of calculation of phase matching angle $\left(\theta_{p m}\right)$, acceptance angle $(\Delta \theta)$, acceptance wavelength $(\Delta \lambda)$ and walk-off angle $(\rho)$ etc. for type-I and type-II SHG are given in Ref. 9. We have also found a simplest method to obtain SHG cut-off wavelength for both type-I and type-II processes and that can be evaluated by solving the following Eqns. 2 and 3, respectively, by using any mathematical software or computer programming,

$$
\begin{gathered}
n_{1}^{o}-n_{3}^{e}=0, \\
n_{1}^{e}+n_{2}^{o}-2 n_{3}^{e}=0,
\end{gathered}
$$

where, $n_{3}^{o}\left(n_{3}^{e}\right)$ and $n_{i}^{o}\left(n_{i}^{e}\right)$ ordinary (extraordinary) refractive indices at the generated and input fundamental wavelengths ( $i=1$ and 2), respectively. The refractive indices have been calculated using the available Sellmeier dispersion relations [5-9] of the respective crystals.

Figures 1 and 2 demonstrate the phase-matching and groupvelocity mismatch characteristics of five potential NLO borate crystals considered for investigation in this work. Solid curves correspond to phase matching and dotted curves show variation of $\Delta_{v g}$. For type-II interaction $\Delta_{v g}$ between the $\lambda_{1}$ and $\lambda_{3}$ waves have been plotted, for example. From Figs. (1) and (2) it is clearly observed that it is always type-I interaction, which permits the generation of shortest VUV wavelengths by SHG. It may also be noted that type-II interaction is not allowed in LB 4 crystal by its crystal symmetry.

Figure 1 shows that only KBBF crystal has the capability of generation of VUV wavelength even below $200 \mathrm{~nm}$, being

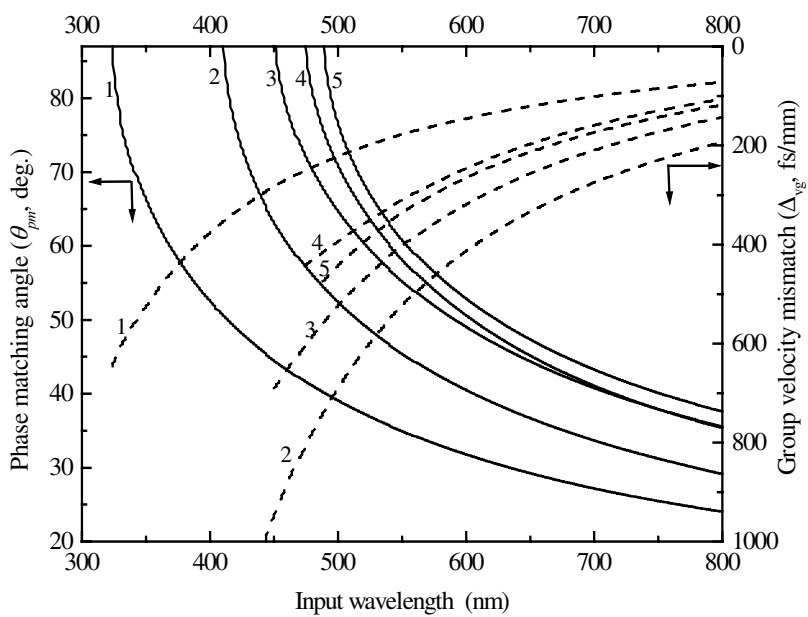

FIG. 1: Phase matching (solid curves) and group-velocity mismatch (dotted) characteristics for type-I SHG in various crystals. Curves marked 1, 2, 3, 4, and 5 corresponds to $\mathrm{KBBF}$ (162), BBO (204.8), KABO (225.5), CLBO (236.8), and LB4 (243.8) crystal, respectively. Bracketed data show the type-I SHG cut-off wavelength (in $\mathrm{nm}$ ) of the respective crystal.

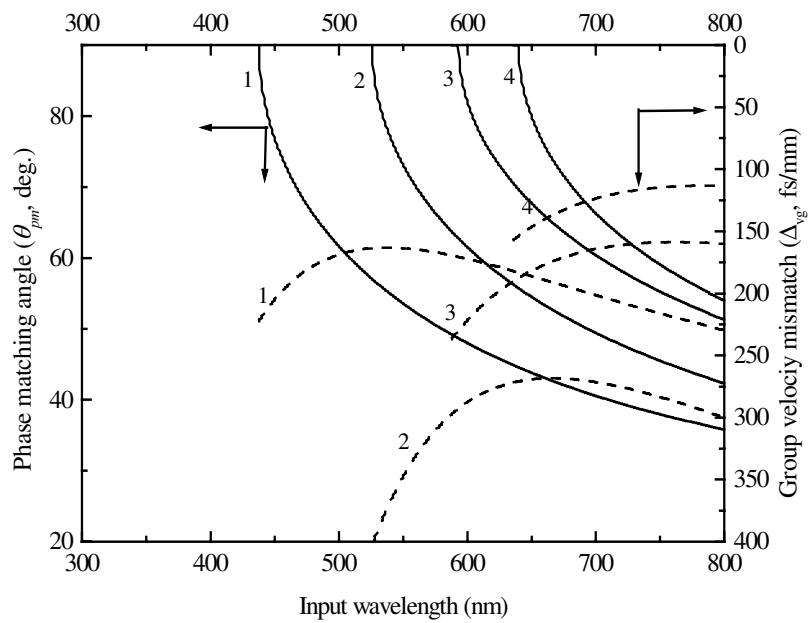

FIG. 2: Phase matching (solid) and group-velocity mismatch (dotted) characteristics for type-II SHG in various NLO borate crystals. Curves marked 1, 2, 3, and 4 correspond to KBBF (224.0), BBO (262.7), KABO (294.6), and CLBO (318.0), respectively. Bracketed data show the type-I SHG cut-off wavelength (in $\mathrm{nm}$ ) of the respective crystal. In LB4 type-II is not allowed.

the type-I SHG cut-off at $162 \mathrm{~nm}$. This is the shortest wavelength that can be generated till date in any crystal by SHG. Reported [8] value of the laser damage threshold (LDT) of $\mathrm{KBBF}$ crystals are as high as $4000 \mathrm{GW} / \mathrm{cm}^{2}$ at $532 \mathrm{~nm}$ with 7 -ns pulse width and $10 \mathrm{~Hz}$ and above $1000 \mathrm{GW} / \mathrm{cm}^{2}$ at 400 $\mathrm{nm}$ with 50 -fs pulse width and $1 \mathrm{kHz}$. However, the problem with this crystal is that its growth is heavily limited by the strong layering tendency [8]. BBO crystal is next to generating SHG wavelength near $200 \mathrm{~nm}$ and also this has the highest value of NLO coefficient for both type-I and type-II SHG 
TABLE I: Some important properties of several NLO crystals.

\begin{tabular}{ccccccccc}
\hline Crystal & $\begin{array}{c}\text { Transmi- } \\
\text { ssion range } \\
(\mathrm{nm})\end{array}$ & $\begin{array}{c}\text { Type-I } \\
\text { SHG } \\
\text { cutoff } \\
(\mathrm{nm})\end{array}$ & $\begin{array}{c}\theta_{\text {pm }} \\
(\mathrm{deg})\end{array}$ & $\begin{array}{c}d_{\text {eff }} \\
(\mathrm{pm} / \mathrm{V})\end{array}$ & $\begin{array}{c}\rho \\
(\mathrm{mrad})\end{array}$ & $\begin{array}{c}\mathrm{L} \Delta \theta \\
(\mathrm{cm}- \\
\mathrm{mrad})\end{array}$ & $\begin{array}{c}\mathrm{L} \Delta \lambda \\
(\mathrm{cm}- \\
\mathrm{nm})\end{array}$ & $\begin{array}{c}L_{\text {eff }}{ }^{*} \\
(\mathrm{~mm})\end{array}$ \\
\hline BBO & $189-3500$ & 204.8 & 29.0 & 2.09 & 67.8 & 0.31 & 0.24 & 0.51 \\
CLBO & $175-2750$ & 236.7 & 36.2 & 0.50 & 34.7 & 0.68 & 0.44 & 0.94 \\
KABO & $180-3600$ & 225.8 & 35.5 & 0.37 & 47.9 & 0.48 & 0.33 & 0.69 \\
KBBF & $155-3780$ & 162.0 & 24.0 & 0.41 & 41.2 & 0.58 & 0.66 & 1.38 \\
LB4 & $170-3300$ & 243.8 & 36.0 & 0.10 & 36.0 & 0.61 & 0.40 & 0.84
\end{tabular}

*For the calculation of $L_{e f f}, T=100 \mathrm{fs}$ has been considered.

as can be seen in Fig. 3. However, growth of good quality large size BBO crystal is not easy and also it is hygroscopic in nature. CLBO crystal has lowest GVM for type-II phasematching interactions and moderate $d_{\text {eff }}$ for both type-I and type-II SHG as is shown in Fig. 3. Growth of CLBO crystal is although comparatively easier, it is highly hygroscopic in nature and requires specific atmospheric conditions for its best performances. KABO and LB4 crystals are nonhygroscopic in nature and particularly LB4 crystal is easily grownable to good quality large sizes. However, the disadvantage of KABO and LB4 crystals are that the value of $d_{e f f}$ is very small and it is lowest for LB4 as is evident in Fig. 3. This can be overcome at least partially by their high value LDT and employing tight focusing geometries. LDT of LB4 is about $40 \mathrm{GW} / \mathrm{cm}^{2}$ at $1064 \mathrm{~nm}$ which is $\sim 3$ times that of BBO $\left(13.5 \mathrm{GW} / \mathrm{cm}^{2}\right)$ and $\sim 1.5$ times that of CLBO $\left(26 \mathrm{GW} / \mathrm{cm}^{2}\right)$. Fig. 4 shows the calculated type-I SHG efficiency, considering the crystal length $(L)=100 \mu \mathrm{m}$, in each case. The solid, dotted, dashed-dotdot, dashed, and dashed-dot curves are for BBO, KABO, LB4, CLBO, and KBBF crystals, respectively. The SHG efficiency have been calculated using infinite plane wave approximation and it is given by $\operatorname{sinc}^{2}(\Delta k L / 2)$ function, where $\Delta k=k_{3}^{e}\left(\theta_{p m}\right)$ $2 k_{i}^{o}$, and $k_{j}$ is the wave vector corresponding to $\lambda_{j}$ radiation. From the Fig. 4 it is observed that the full widths of the curves at half maximum (FWHM) level of SHG efficiency are 13, 17, 21, 23, and $37 \mathrm{~nm}$, for BBO, KABO, LB4, CLBO, and KBBF crystals, respectively, centered at $600 \mathrm{~nm}$. During the calculations the refractive indices were calculated using the available Sellmeier dispersion relations $[5,6]$ of the respective crystals. The SHG phase-matching bandwidth of KBBF crystal is 37 $\mathrm{nm}$, which is the highest among all the crystals.

\section{ULTRAFAST LASER PULSE MEASUREMENT AND SHG CRYSTAL}

With the recent development in solid-state mode-locked laser technology the generation of ultrafast laser pulses of

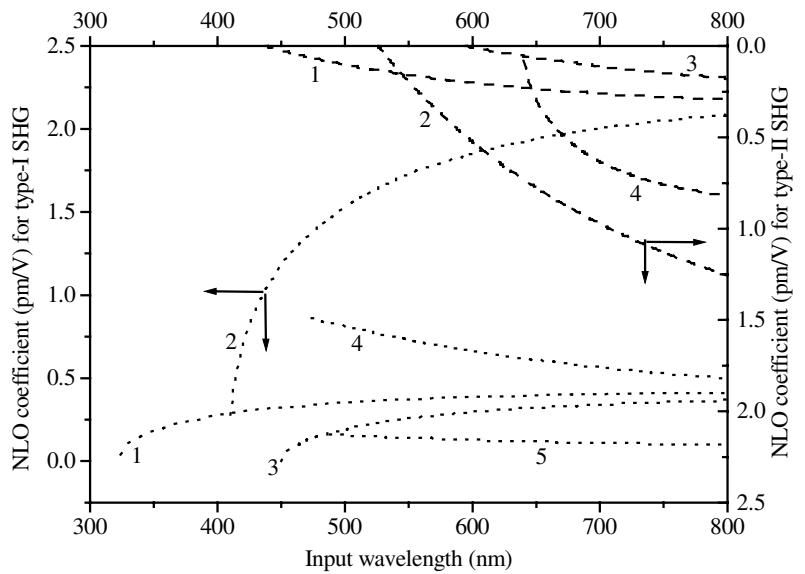

FIG. 3: Effective NLO coefficients for type-I (dotted) and type-II (dashed) SHG interactions in various borate group NLO crystals. The curves marked 1, 2, 3, 4, and 5 are for KBBF, BBO, KABO, CLBO, and LB4 crystals, respectively.

few fs (say, 4 fs) duration is now a routine technique. A breakthrough in the full characterization of ultrashort pulses occurred few years ago with the introduction of frequencyresolved optical gating (FROG) [10,11]. FROG measures a two-dimensional (2-D) spectrogram in which the signal of any autocorrelation-type experiment is resolved as a function of both time delay and frequency [11]. The full pulse intensity and phase may be subsequently retrieved from such a spectrogram (called FROG trace) via an iterative retrieval algorithm. The determination of the pulse structure and the phase information of such few cycle laser pulses is very much important since these plays a decisive role in many applications [10]. In general, the FROG pulse reconstruction does not depend on pulse duration since the FROG traces simply scale in the time-frequency domain. However, with the decrease of the pulse duration that is accompanied by the increase in the spectral bandwidth, the experimentally collected data be- 


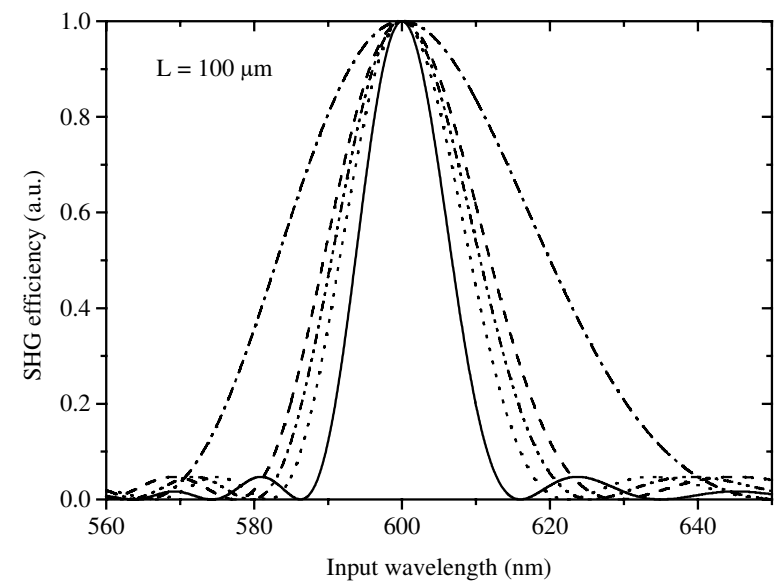

FIG. 4: Conversion efficiency (under plane-wave approximation) for type-I SHG of broadband ultrafast laser radiation in different NLO crystals $(L=100 \mu \mathrm{m})$. The solid, dotted, dashed-dot-dot, dashed, and dashed-dot curves are for BBO (13), KABO (17), LB4 (21), CLBO (23), and KBBF (37) crystals, respectively. Inside the bracket the FWHM (in $\mathrm{nm}$ ) of the respective curves have been shown.

gin to deviate significantly from the mathematically defined ideal FROG trace. In a FROG pulse reconstruction process the limited SHG spectral bandwidth of the NLO crystal become increasingly important for $<10$-fs pulses. Therefore, it is highly desirable that the used SHG crystal will have higher SHG spectral bandwidth. Thus the newly developed KBBF crystal, which has highest SHG spectral bandwidth, may take very important role, if it is introduced in a FROG experiment. In addition, KBBF crystal has very high value of laser damage threshold, and it is transparent even down to $155 \mathrm{~nm}$ in VUV, which are also very useful characteristics of a NLO material.

\section{CONCLUSIONS}

In conclusion, we have presented several NLO parameters for generation of second harmonic of ultrafast femtosec- ond laser radiation by employing type-I and type-II phasematching in various NLO crystals including some recently discovered crystals, whose potentialities for use in femtosecond experiments are yet to be verified conclusively. For generation of second harmonic radiation by employing birefringent phase-matching technique in the widely used BBO crystal, the angular and wavelength sensitivity, and the spatial walkoff angle are highest in comparison to those of other crystals. These properties may have detrimental effects in some NLO experiments. Another crystal, CLBO is highly hygroscopic which limits its practical application although it has several other advantageous characteristics. Comparatively newly discovered KABO and LB4 crystals have good mechanical properties and high value of LDT [6,7]. However, these materials suffer due to their low NLO coefficient. A recently discovered crystal, KBBF has moderate walk-off angle, and wider spectral and angular bandwidths. It is found that the value of type-I SHG spectral bandwidth $(\Delta \lambda)$ of KBBF crystal is the highest and $\Delta \lambda$ for $100 \mu \mathrm{m}$ thick BBO, KABO, LB4, CLBO, and $\mathrm{KBBF}$ crystals are $13,17,21,23$, and $37 \mathrm{~nm}$, respectively for generation of second harmonic of a broadband fundamental radiation, centered at $600 \mathrm{~nm}$. The LDT of KBBF crystal is $4000 \mathrm{GW} / \mathrm{cm}^{2}$ at $532 \mathrm{~nm}$ with 7-ns pulse and $10 \mathrm{~Hz}$ rep. rate and $1000 \mathrm{GW} / \mathrm{cm}^{2}$ at $400 \mathrm{~nm}$ with 50 -fs pulse and $1 \mathrm{KHz}$ rep. rate [8]. These properties of KBBF may be very attractive in different NLO applications viz. for characterization of high power ultrashort laser pulses with ultra-wide bandwidth. Moreover, utilizing the wide spectral acceptance it may be possible to achieve generation of short pulse UV radiation in KBBF crystal, by frequency doubling the broadband visiblenear infrared femtosecond laser radiation, say, the output from a optical parametric amplifier $[3,4]$. However, we hope that the presented results will bring a lot of interest among the researchers to use the newly discovered crystalline materials in different NLO experiments.

\section{Acknowledgement}

PK is grateful to Prof. T. Kobayashi, Tokyo University, Japan, for his suggestions during the visit of PK to the Tokyo University. Authors are grateful to NIT Durgapur for providing the computational facilities.
[1] A. Shirakawa and T. Kobayashi, IEICE Trans. Electron. E81-C, 246 (1998).

[2] G. Cerullo and S. De. Silvestri, Rev. Sci. Instrum. 74, 1 (2003).

[3] A. Baltuska and T. Kobayashi, Appl. Phys. B Lasers Opt. B75, 427 (2002).

[4] S. Adachi, P. Kumbhakar, and T. Kobayashi, Opt. Lett. 29, 1150 (2004).

[5] Y. Mori, I. Kuroda, S. Nakajima, T. Sasaki, and S. Nakai, Appl. Phys. Lett. 67, 1818 (1995).

[6] Z. Hu, T. Higashiyama, M. Yoshimura, Y. K. Yap, Y. Mori, and
T. Sasaki, Jpn. J. Appl. Phys. 37, L1093 (1998).

[7] T. Sugawara, R. Komatsu, and S. Uda, Solid State. Commun. 107, 233 (1998).

[8] C. Chen, Z. Lin, and Z. Wang, Appl. Phys. B. Lasers Opt. B80, 1 (2005).

[9] V. G. Dmitriev, G. G. Gurzadyan, and D. N. Nikogosyan, Handbook of nonlinear optical crystals. Springer, Heidelberg, 1999, Ch. 2. 